\title{
Alterations in Rev-ERBa/BMAL1 ratio and glycated hemoglobin in rotating shift workers: the EuRhythDia study
}

\author{
Stefano Rizza ${ }^{1}$ - Alessio Luzi ${ }^{1}$. Maria Mavilio ${ }^{1} \cdot$ Marta Ballanti $^{1} \cdot$ Arianna Massimi $^{1}$. Ottavia Porzio ${ }^{2}$. \\ Andrea Magrini ${ }^{3}$. Juliane Hannemann ${ }^{4} \cdot$ Rossella Menghini $^{1} \cdot$ Michael Lehrke $^{5} \cdot$ Bart Staels $^{6} \cdot$ Peter J. Grant ${ }^{7}$. \\ Rainer H. Boger ${ }^{4}$. Nikolaus Marx ${ }^{5}$. Massimo Federici ${ }^{1}$ (I)
}

Received: 30 November 2020 / Accepted: 9 January 2021 / Published online: 31 March 2021

(c) The Author(s) 2021, corrected publication 2021

\begin{abstract}
Objective To detect premature gluco-metabolic defects among night shift workers with disturbances in circadian rhythms. Design and methods We performed a hypothesis-generating, cross-sectional analysis of anthropometric, metabolic, lipid, and inflammation parameters, comparing active (a-NSW, $n=111$ ) and former (f-NSW, $n=98$ ) rotating night shift workers with diurnal workers (controls, $n=69$ ). All participants were hospital nurses. We also evaluated the Pittsburgh Sleep Quality Index (PSQI) and assessed expression of transcription factors REV-ERB $\alpha$ and BMAL1 in peripheral blood mononuclear cells (PBMCs), as indicators of the molecular clock.

Results Both a-NSW and f-NSW participants had significantly higher glycated hemoglobin (HbA1c) and white blood cell counts (WBC) $(p<0.001$ for both), PSQI global score $(p=0.001)$ and diastolic blood pressure levels $(p=0.024)$ compared with controls. Expression of REV-ERB $\alpha /$ BMAL1 RNA in PBMC was significantly higher in a-NSW $(p=0.05)$ than in f-NSW or control participants. Multivariate regression analysis showed that working status and PSQI were independent determinants of higher HbA1c levels $(p<0.001)$.

Conclusions We demonstrated that young, healthy night shift workers show subclinical abnormalities in HbA1c and changes in peripheral clock gene expression.
\end{abstract}

Keywords Diabetes $\cdot$ Insulin resistance $\cdot$ Circadian $\cdot$ Obesity

Managed by Antonio Secchi.

Massimo Federici

federicm@uniroma2.it

1 Department of Systems Medicine, University of Rome Tor Vergata, Via Montpellier, 100133 Rome, Italy

2 Department of Experimental Medicine, University of Rome Tor Vergata, Rome, Italy

3 Department of Biomedicine and Prevention, University of Rome Tor Vergata, Rome, Italy

4 Institute of Clinical Pharmacology and Toxicology, University Medical Center Hamburg-Eppendorf, Hamburg, Germany

5 Department of Cardiology, University Medical Center Aachen, Aachen, Germany

6 Univ. Lille, INSERM, CHU Lille, Institut Pasteur de Lille, U1011 - EGID, F-59000 Lille, France

7 Leeds Institute of Cardiovascular and Metabolic Medicine, University of Leeds, Leeds, England

\author{
Abbreviations \\ NSW Night shift work \\ PSQI Pittsburgh Sleep Quality Index \\ PBMCs Peripheral blood mononuclear cells \\ HbA1c Glycated hemoglobin
}

\section{Introduction}

Night shift work (NSW) is highly prevalent in Western societies, with up to $20 \%$ of the European working population engaged in some type of shift work schedule [1], increasing to $45 \%$ in the healthcare sector [1]. Recently, epidemiological studies have suggested that rotating NSW is associated with a higher risk of developing type 2 diabetes (T2D) and cardiovascular diseases [2, 3]. Furthermore, even when diet is controlled, night shift workers exhibit poorer metabolic health than daytime workers [4]. Night shift workers, for example, exhibit higher plasma triglyceride levels [5], which is related to circadian system disruption; 
postprandial glucose and lipid tolerance to standard test meals are impaired after switching to NSW [6]. In addition, disrupted glucose metabolism and circadian misalignment during short sleep have been reported repeatedly [7, 8]. In the Nurse Health Study II, an increased risk of T2D was observed in participants with sleep difficulties doing rotating night shifts [9]. Moreover, in patients with overt diabetes, there is an association between glycemic control and sleep disturbances, while impaired melatonin secretion, insufficient duration and sleep quality have an adverse effect on insulin sensitivity and metabolic risk factors [10-12].

We conducted a hypothesis-generating, cross-sectional analysis of metabolic and circadian rhythm biomarkers in a group of hospital nurses recruited from the same working environment. Our results suggest that NSW in apparently healthy individuals is associated with subclinical defects that are typically manifested in people at risk of developing chronic metabolic disorders.

\section{Methods}

\section{Participants}

A group of volunteer hospital nurses was recruited at the Policlinico Tor Vergata University between 2012 and 2015 as part of the EuRhythDia project. The EuRhythDia consortium set out to investigate the disruption of the circadian clock in rotating night shift workers. A written consent was obtained from each patient after full explanation of the purpose and nature of all study procedures used. Participants received detailed information about the study protocol and, after providing written consent, underwent clinical examination and a standardized interview. Exclusion criteria included the presence of diabetes, liver disease, renal insufficiency, heart failure, coagulopathy or any other severe systemic disease. Subjects were also excluded if they had a history of any form of cancer or if they had positive blood tests for HIV, hepatitis B or hepatitis C or if they had taken melatonin supplements within four weeks before commencing the study.

In women, the study was initiated during the early follicular phase of the menstrual cycle.

Hospital workers were eligible if they had been working for a minimum of 2 years. We divided hospital nurses into three groups: [1] active rotating night shift workers (a-NSW, $n=111$ ), working a shift schedule of from four to seven 12-h nights per month, followed by 2 days off; [2] former rotating night shift workers (f-NSW, $n=98$ ), who had stopped night shift working for at least 2 years before inclusion in the study; and [3] daily workers (controls, $n=69$ ): nurses who had never worked night shifts. In the overall population, body mass index (BMI) was calculated by dividing the weight (in kilograms) by the square of height (in meters). Three blood pressure measurements were obtained in the dominant arm in the sitting position and then averaged to calculate mean systolic- and diastolic-BPs. Current and former smokers were considered together as a single group and compared to never smokers.

\section{Clock genes analysis}

After an overnight fast, blood samples and anthropometric parameters were obtained for controls and f-NSW between 8:00 and 9:00 AM whereas after $12 \mathrm{~h}$ of night shift while fasting conditions in a-NSW. About $20 \mathrm{ml}$ whole blood was collected from each subject. Of these, about $8 \mathrm{~mL}$ was used for extraction of PMBC RNA and real-time quantitative RTPCR analysis, as previously described [11]. Briefly, singlestrand cDNA was synthesized, according to the Applied Biosystems (Foster City, CA) standard protocol, from $2 \mu \mathrm{g}$ of total RNA sample using High-Capacity cDNA Archive Kit. Fifty nanograms of cDNA was amplified by real-timePCR RNA expression of clock (BMAL1 and REV-ERB $\alpha$ ), inflammatory (IL-1 $\beta$, IF- $\gamma$ ) using an ABI PRISM 7500 System and normalized to $18 \mathrm{~S}$ rRNA as an endogenous control [13].

\section{Sleep quality analysis}

The Pittsburgh Sleep Quality Index (PSQI) is a validated and widely used scale to identify elements of sleep over the past 30 days and reflects the individual's perception of sleep during shift and non-shift days [14].

This questionnaire identifies seven "components" of sleep routinely assessed clinically: sleep latency, sleep duration, habitual sleep efficiency, sleep disturbance, use of sleep medication, daytime dysfunction and subjective sleep quality. The sum of scores of the aforementioned seven components gives a global PSQI score that ranges from 0 to 21 points; a score of 5 or greater is associated with poor sleep quality and was therefore dichotomized as such in our analysis. The PSQI scores have been shown to have good test-retest reliability, with a correlation coefficient of 0.85 for the global score, and correlation coefficients ranging from 0.65 (medication use) to 0.84 (sleep latency) for the component scores $[14,15]$.

\section{Statistical analysis}

Participants' clinical characteristics were reported as means and standard deviations or as frequencies and percentages for continuous and categorical variables, respectively. Each continuous variable was checked for normality of distribution by the Kolmogorov-Smirnov test. 
Univariate ANOVA was used to compare each of the reported variables in the a-NSW, f-NSW and control groups as described [16]. The Mann-Whitney test was used for variables with non-normal distribution.

The significance of difference between percentages in groups was evaluated by using the $\chi^{2}$ test. We used Spearman's rho coefficient to calculate a nonparametric measure of rank correlation between PSQI score, glucose and glycated hemoglobin ( $\mathrm{HbA} 1 \mathrm{c})$. We used linear regression analysis to explore independent associations between $\mathrm{HbA1c}$ entered into the model as the dependent variable and PSQI and working status as possible predictors controlled for age, sex and body mass index (BMI).

A $p$-value $<0.05$ was considered statistically significant. All analyses were performed using SPSS for Windows software version 19.0 (IBM Corp., Armonk, NY, USA).

\section{Results}

Anthropometric, clinical, metabolic and inflammatory characteristics of the study population are shown in Table 1 . None of the participants had diabetes or other chronic metabolic or cardiovascular diseases. Participants in the a-NSW group $(n=111)$ were significantly younger $(p<0.001)$ than controls $(n=69)$ and f-NSW $(n=98)$, whereas the three groups did not differ significantly with respect to sex, lipid profile, presence of metabolic syndrome or numbers of smokers. Moreover, the groups were comparable regarding non-modifiable risk factors such as family history of diabetes. Mean HbA1c values were significantly higher in the a-NSW and f-NSW groups than in controls $(p<0.001)$, even after adjustment for BMI, age and sex (Table 1). Participants in a-NSW, compared with those in f-NSW and controls, had significantly higher BMI $(p=0.013)$ and creatinine $(p=0.038)$. No difference in systolic blood pressure
Table 1 Anthropometric, clinical, sleep, metabolic and inflammatory characteristics of the study population divided by working status: day workers (DW), active night shift workers (a-NSW) and former night shift workers (f-NSW)

\begin{tabular}{|c|c|c|c|c|}
\hline Variables & DW $(n=69)$ & a-NSW $(n=111)$ & f-NSW $(n=98)$ & $p$ \\
\hline Age (years) & $37.0 \pm 7.2$ & $36.1 \pm 6.0$ & $39.7 \pm 7.1$ & $<0.001$ \\
\hline BMI & $23.7 \pm 3.5$ & $24.8 \pm 3.7$ & $25.7 \pm 4.6$ & 0.013 \\
\hline Gender (f/m) & $39 / 25$ & $74 / 37$ & $72 / 26$ & 0.236 \\
\hline Metabolic syndrome (y/n) & $1 / 63$ & $10 / 101$ & $6 / 92$ & 0.145 \\
\hline Smoker $(y / n)$ & $19 / 45$ & $50 / 61$ & $36 / 62$ & 0.120 \\
\hline T2D first-degree relatives $(\mathrm{y} / \mathrm{n})$ & $23 / 41$ & $33 / 78$ & $37 / 61$ & 0.444 \\
\hline $\mathrm{SBP}(\mathrm{mmHg})$ & $112.0 \pm 12.0$ & $114.0 \pm 12.8$ & $112.0 \pm 13.3$ & 0.450 \\
\hline $\mathrm{DBP}(\mathrm{mmHg})$ & $71.0 \pm 9.5$ & $74.8 \pm 9.3$ & $73.6 \pm 8.4$ & 0.024 \\
\hline Fasting glucose (mg/dL) & $89.7 \pm 10.7$ & $88.7 \pm 8.1$ & $89.9 \pm 8.2$ & 0.571 \\
\hline Fasting insulin $(\mu \mathrm{U} / \mathrm{mL})$ & $10.4 \pm 5.4$ & $12.8 \pm 14.3$ & $12.0 \pm 9.6$ & 0.391 \\
\hline HOMA-IR & $2.35 \pm 1.37$ & $2.63 \pm 2.12$ & $2.68 \pm 2.24$ & 0.566 \\
\hline HOMA-beta & $149.2 \pm 125.6$ & $180.3 \pm 170.9$ & $174.8 \pm 145.8$ & 0.399 \\
\hline HbA1c $(\%)$ & $5.12 \pm 0.24$ & $5.32 \pm 0.32$ & $5.33 \pm 0.30$ & $<0.001 *$ \\
\hline Creatinine $(\mathrm{mg} / \mathrm{dL})$ & $0.79 \pm 0.15$ & $0.82 \pm 0.15$ & $0.77 \pm 0.13$ & 0.038 \\
\hline Total cholesterol (mg/dL) & $190.2 \pm 36.5$ & $189.7 \pm 34.8$ & $197.7 \pm 37.0$ & 0.231 \\
\hline HDL cholesterol (mg/dL) & $58.5 \pm 13.9$ & $59.4 \pm 16.6$ & $56.4 \pm 15.7$ & 0.364 \\
\hline LDL cholesterol (mg/dL) & $112.2 \pm 33.2$ & $110.4 \pm 33.1$ & $121.1 \pm 33.0$ & 0.047 \\
\hline Triglycerides $(\mathrm{mg} / \mathrm{dL})$ & $97.4 \pm 65.4$ & $99.4 \pm 67.7$ & $101.3 \pm 66.7$ & 0.934 \\
\hline WBC count $\left(\times 10^{3} / \mathrm{mL}\right)$ & $5.7 \pm 1.2$ & $6.9 \pm 1.9$ & $6.6 \pm 1.7$ & $<0.001$ \\
\hline $\mathrm{CRP}(\mathrm{mg} / \mathrm{L})$ & $1.4 \pm 2.8$ & $1.7 \pm 3.0$ & $2.1 \pm 3.2$ & 0.309 \\
\hline PSQI global score (score 1-4/5-21) & $51 / 13$ & $54 / 57$ & $43 / 55$ & $0.001 *$ \\
\hline$R E V-E R B \alpha$ mRNA & $1.298 \pm 1.66$ & $1.230 \pm 0.626$ & $1.218 \pm 0.584$ & 0.861 \\
\hline$B M A L 1$ mRNA & $4.907 \pm 6.693$ & $4.170 \pm 2.863$ & $4.520 \pm 2.804$ & 0.506 \\
\hline$R E V-E R B \alpha / B M A L 1$ & $0.310 \pm 0.164$ & $0.369 \pm 0.210$ & $0.318 \pm 0.158$ & 0.050 \\
\hline IFN- $\gamma$ mRNA & $0.74 \pm 0.60$ & $0.84 \pm 0.87$ & $0.99 \pm 1.1$ & 0.208 \\
\hline IL- $1 \beta$ mRNA & $1.74 \pm 3.91$ & $8.94 \pm 20.31$ & $8.70 \pm 20.40$ & 0.014 \\
\hline
\end{tabular}

*Adjusted for age, sex and BMI

BMI, body mass index; T2D, type 2 diabetes; SBP, systolic blood pressure; DBP, diastolic blood pressure; HOMA-IR, homeostatic model assessment of insulin resistance; HbA1c, glycated hemoglobin; HDL, highdensity lipoprotein; LDL, low-density lipoprotein; WBC, white blood cells; CRP, C-reactive protein; PSQI, Pittsburgh Sleep Quality Index questionnaire; IFN- $\gamma$, interferon- $\gamma$; IL- $1 \beta$, interleukin- $1 \beta$, 
(BP) was observed between groups (Table 1), whereas both a-NSW and f-NSW had significantly higher diastolic BP levels ( $p=0.024)$, white blood cell (WBC) count $(p<0.01)$ and serum interleukin (IL)-1 $\beta$ levels $(p=0.014)$ compared with controls. Interestingly, diastolic, but not systolic, BP was significantly correlated with age $(r=0.129, p=0.029)$. Mean values of metabolic parameters were similar, including fasting glucose and the degree of insulin resistance as measured using the homeostatic model assessment of insulin resistance (HOMA-IR). HbA1c was significantly correlated with WBC count $(r=0.153, p=0.013)$ and with IL-1 $\beta$ ( $r=0.138, p=0.026)$ in PBMCs. Significant differences in the mean PSQI global score, a marker of sleep quality and circadian alignment, were observed, with a PSQI score $>5$ in $20 \%$ of controls, in $51 \%$ of $\mathrm{f}-\mathrm{NSW}$ workers and in $56 \%$ of a-NSW workers $(p=0.001)$ (Table 1). This difference persisted after adjusting for age, sex and BMI. Interestingly, the PSQI score was significantly correlated with HbA1c $(r=0.295, p<0.001)$ but not with fasting glucose $(r=-0.48, p=0.420)$.
Next, we dichotomized the entire population in bad sleepers $(\mathrm{BS}, \mathrm{n}=110)$ and good sleepers $(\mathrm{GS}, n=163)$ based on PSQI score cutoff $>5$ (Table 2 ). BS were slightly older $(p=0.026)$ and more frequently female $(p<0.001)$. Interestingly, HbA1c level was the only metabolic variable significantly different between the groups, with a higher HbA1c in BS compared to GS ( $p=0.025)$ even after adjustment for age, sex and BMI. Of note, the REV-ERB $\alpha / B M A L 1$ ratio was significantly lower in GS $(p<0.049)$. PSQI significantly correlated with HbA1c but not with fasting glucose (Fig. 1).

Then, we looked for markers of the positive and negative control of circadian clock in PBMCs. We observed no significant difference in leukocyte clock gene expression among the three groups $(p=0.506$ for BMAL1 and $p=0.861$ for $R E V-E R B \alpha)$. Although individual gene expression levels did not differ, we found that the $R E V-E R B \alpha / B M A L 1$ ratio was significantly higher in a-NSW than in f-NSW and control participants $(p=0.023)$. Of note, among all study covariates, $R E V-E R B \alpha / B M A L 1$ ratio expression was significantly correlated only with diastolic BP $(r=0.133, p=0.024)$.
Table 2 Anthropometric, clinical, metabolic and inflammatory characteristics of the study population divided upon sleep quality

\begin{tabular}{|c|c|c|c|}
\hline Variables & Good sleeper $(n=163)$ & Bad sleeper $(n=110)$ & $p$ \\
\hline Age (years) & $36.9 \pm 6.5$ & $38.7 \pm 6.3$ & 0.026 \\
\hline BMI & $24.9 \pm 3.8$ & $24.9 \pm 4.4$ & 0.992 \\
\hline Gender (f/m) & $97 / 66$ & $88 / 22$ & $<0.001$ \\
\hline Metabolic Syndrome (y/n) & $153 / 10$ & $103 / 7$ & 0.565 \\
\hline Smoker $(y / n)$ & $98 / 65$ & $70 / 40$ & 0.324 \\
\hline T2D first-degree relatives $(y / n)$ & $113 / 50$ & $67 / 43$ & 0.096 \\
\hline $\mathrm{SBP}(\mathrm{mmHg})$ & $112.6 \pm 12.9$ & $113.1 \pm 12.8$ & 0.739 \\
\hline $\mathrm{DBP}(\mathrm{mmHg})$ & $72.8 \pm 8.7$ & $74.5 \pm 9.8$ & 0.122 \\
\hline Fasting glucose (mg/dl) & $89.4 \pm 7.8$ & $89.2 \pm 10.2$ & 0.866 \\
\hline Fasting Insulin $(\mu \mathrm{U} / \mathrm{ml})$ & $11.6 \pm 11.1$ & $12.5 \pm 11.2$ & 0.515 \\
\hline HOMA-IR & $2.43 \pm 1.52$ & $2.80 \pm 2.57$ & 0.141 \\
\hline HOMA-beta & $156.9 \pm 132.6$ & $191.9 \pm 187.8$ & 0.088 \\
\hline $\mathrm{HbA} 1 \mathrm{c}(\%)$ & $5.23 \pm 0.29$ & $5.33 \pm 0.32$ & $0.025 *$ \\
\hline Creatinine (mg/dl) & $0.79 \pm 0.14$ & $0.79 \pm 0.14$ & 0.864 \\
\hline Total cholesterol (mg/dl) & $190.2 \pm 34.0$ & $196.5 \pm 38.8$ & 0.159 \\
\hline HDL cholesterol (mg/dl) & $56.3 \pm 15.5$ & $60.8 \pm 15.6$ & 0.019 \\
\hline LDL cholesterol (mg/dl) & $114.3 \pm 31.5$ & $115.2 \pm 34.0$ & 0.831 \\
\hline Triglycerides (mg/dl) & $97.8 \pm 67.0$ & $102.2 \pm 71.9$ & 0.591 \\
\hline $\mathrm{WBC}\left(\times 10^{3} / \mathrm{ml}\right)$ & $7.04 \pm 1.52$ & $6.22 \pm 1.50$ & 0.103 \\
\hline C-reactive protein $(\mathrm{mg} / \mathrm{l})$ & $1.43 \pm 1.16$ & $2.02 \pm 2.1$ & 0.548 \\
\hline REV-ERB $\alpha$ mRNA & $1.27 \pm 0.46$ & $1.25 \pm 1.0$ & 0.944 \\
\hline BMAL1 mRNA & $4.59 \pm 4.40$ & $5.30 \pm 4.9$ & 0.658 \\
\hline REV-ERB $\alpha / B M A L 1$ & $0.4 \pm 0.1$ & $0.3 \pm 0.1$ & 0.049 \\
\hline IFN-gamma & $0.8 \pm 0.8$ & $0.9 \pm 1.0$ & 0.685 \\
\hline IL-1 $\beta$ & $7.3 \pm 20.0$ & $6.77 \pm 14.2$ & 0.824 \\
\hline
\end{tabular}

BMI, body mass index; T2D, type 2 diabetes; SBP, systolic blood pressure; DBP, diastolic blood pressure; HOMA-IR, homeostatic model assessment of insulin resistance; HbA1c, glycated hemoglobin; HDL, highdensity lipoprotein; LDL, low-density lipoprotein; WBC, white blood cells; CRP, C-reactive protein; PSQI, Pittsburgh Sleep Quality Index questionnaire; IFN- $\gamma$, interferon- $\gamma$; IL- $1 \beta$, interleuchin- $1 \beta$ 

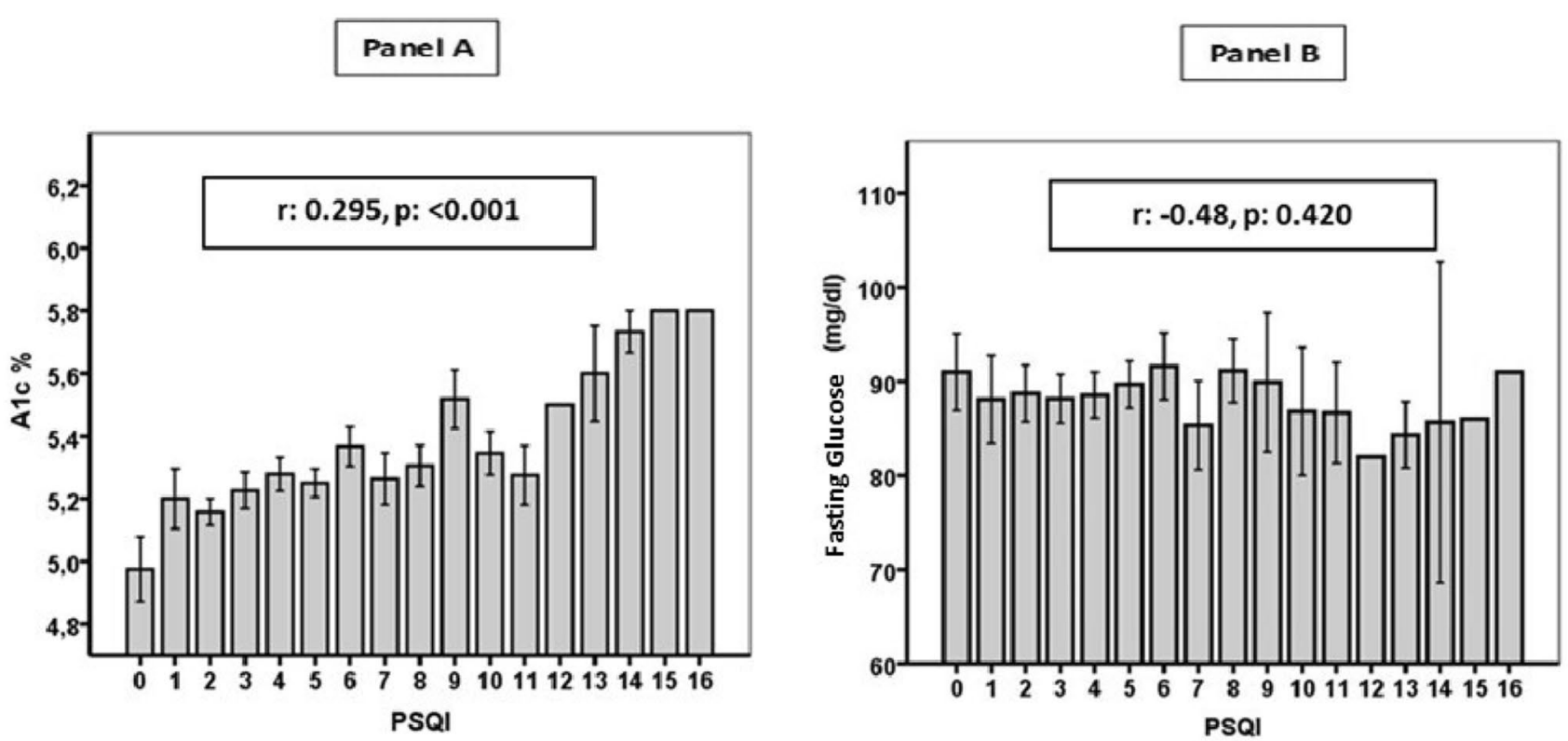

Fig. 1 Correlation between Pittsburgh Sleep Quality Index (PSQI) and glycated hemoglobin (HbA1c, panel A), and PSQI and fasting glucose (panel B). Error bars indicate standard deviations

Table 3 Multivariate linear regression with glycated hemoglobin (HbA1c) as dependent variable $\left(R^{2}: 0.165, p<0.001\right)$

\begin{tabular}{lrrr}
\hline Variable & \multicolumn{1}{c}{ Beta } & 95\% CI for beta & \multicolumn{1}{l}{$p$} \\
\hline Age (years) & 0.008 & 0.002 to 0.013 & 0.005 \\
Sex (male) & -0.034 & -0.112 to 0.045 & 0.398 \\
BMI & 0.015 & 0.006 to 0.024 & 0.001 \\
WBC count & 0.021 & -0.001 to 0.042 & 0.063 \\
PSQI & 0.127 & 0.054 to 0.200 & 0.001 \\
Working status & 0.053 & 0.006 to 0.101 & 0.028 \\
$\quad$ (a-NSW) & & & \\
\hline
\end{tabular}

BMI, body mass index; WBC, white blood cells; PSQI, Pittsburgh Sleep Quality Index questionnaire; a-NSW, active night shift workers

Multivariate regression analyses showed that both working status and PSQI were independent determinants of higher HbA1c levels, even after controlling for age, sex, BMI and a generic inflammation parameter such as WBC count. The model was highly significant $(F=9.7, p<0.001)$ and explained $16.5 \%$ (adjusted $\mathrm{R}^{2}$ ) of the variance of $\mathrm{HbA} 1 \mathrm{c}$ (Table 3).

\section{Discussion}

In this study, we observed that apparently healthy, young nurses doing rotating night shifts showed mild but significant increases in $\mathrm{HbA} 1 \mathrm{c}$ levels compared with diurnal workers, independently of sex, age and BMI. HbA1c levels were also higher in f-NSW compared with diurnal workers. It is unclear whether the increase in $\mathrm{HbA} 1 \mathrm{c}$ in $\mathrm{f}-\mathrm{NSW}$ derived from some form of metabolic memory or whether NSW had changed lifestyle habits that were maintained after workers ceased NSW. However, our study shows that even relatively young healthy hospital workers, being or having been on night shift, in the absence of important cardiometabolic risk factors, show subclinical metabolic alterations that may be predictive of progression to more severe conditions such as diabetes.

The association of working status with $\mathrm{HbA} 1 \mathrm{c}$, but not with fasting glucose and HOMA-IR may be due to the increased glucose excursions during post-prandial states that might affect the hemoglobin glycation process. Consistently, it has been reported in humans that deregulated feeding and breakfast skipping may alter the function of the central and peripheral circadian clock and, as a result, impair glucose metabolism [17-19]. Similarly, circadian misalignment has been shown to increase inflammation in chronic shift workers [20]. Accordingly, since in study regression model WBC partly explained the increase of $\mathrm{HbA1c}$, we tested the hypothesis that clock genes may be misaligned in PBMCs of a-NSW, which could contribute to increased inflammation [21].

Our data, showing an increase in expression of $\mathrm{IL}-1 \beta$ RNA in PBMCs and high WBC count in a-NSW, and a significant correlation of HbA1c with leukocyte IL-1 $\beta$ RNA expression, suggest a potential common underlying mechanism for these changes and confirm the chronic low-grade inflammation is a recognized key feature associated with the risk of T2D development [22] and its complications [23]. 
Interestingly, we found that the REV-ERB $\alpha / B M A L 1$ mRNA ratio was significantly higher in a-NSW compared with f-NSW and control participants. Actually, REV$E R B \alpha / B M A L 1$ mRNA ratio might be a potential indicator of desynchronization of the master clock caused by aberrant and prolonged artificial light exposure during night shifts [24-27]. However, even if the connection between the circadian clock and metabolism is well established [28], our results do not indicate whether the unbalanced $R E V-E R B \alpha / B M A L 1$ ratio derives from a disturbance of glucose metabolism or represents a marker of the proinflammatory state of circulating monocytes.

Finally, although sleep deprivation and populationbased epidemiological studies have shown that disrupted sleep is a risk factor for hypertension, we observed a significant increase only in diastolic, not systolic, BP in a-NSW compared with the other two groups. However, considering the young mean age of our study population and the significant correlation of age with diastolic BP, our results seem to be consistent with emerging data indicating that the adverse cardiovascular consequences of disrupted sleep may begin during early adulthood [29, 30].

Our work has limitations, including its cross-sectional design and lack of prospective analysis. Moreover, the blood sampling time point after a night shift for a-NSWs may have confounded some variables that vary across the day, including BP, fasting glucose, WBC count, gene expression of $R E V-E R B \alpha / B M A L 1$, but not HbA1c level. Furthermore, the $R E V-E R B \alpha / B M A L 1$ ratio as biomarker of circadian rhythm should be assessed in other cohorts with samples that are available from several time points during the $24 \mathrm{~h}$.

Future studies are warranted to understand how long it may take to increase the glycemic-inflammatory burden in night shift workers and to design appropriate strategies to dampen this effect.

\begin{abstract}
Author contributions S.R., A.L., M.B. and A.M. performed the clinical protocols; M.M., A.M., R.M. and O.P. performed clinical chemistry and mRNA analysis; J.H., B.S., P.J.G., R.H.B., M.F. and N.M. designed the study and provided funding; S.R. performed statistical analysis and drafted the manuscript; A.M., B.S., P.J.G., R.H.B., M.F. and N.M. revised the final full text. M.F. is the guarantor of this work.
\end{abstract}

Funding Open Access funding provided by Università degli Studi di Roma Tor Vergata within the CRUI-CARE Agreement. This work was funded by the European Union's Seventh Framework Programme for research, technological development and demonstration under grant agreement no 278397 (EuRhythDia; Targeting chronotherapeutic lifestyle intervention for diabetes and obesity to reset the circadian rhythm and improve cardiometabolic risk in the European working population) to R.H.B., N.M., B.S., M.F., P.J.G., and by Ministry of University Progetti di Ricerca di Interesse Nazionale (PRIN 2015MPESJS_004 and 2017FM74HK) to M.F. M.L. and N.M. are funded by the Deutsche Forschungsgemeinschaft (DFG, TTR 219, M-03, M-05).

\section{Compliance with ethical standards}

Conflict of interest The authors have no financial or other relevant conflicts of interest to disclose.

Ethical approval All procedures performed in studies involving human participants were in accordance with the ethical standards of the institutional and/or national research committee and with the 1964 Helsinki Declaration and its later amendments or comparable ethical standards. The study was approved by the Comitato Etico Policlinico Tor Vergata n. 56/12 and n. 125/13.

Informed consent Informed consent was obtained from all individual participants included in the study.

Open Access This article is licensed under a Creative Commons Attribution 4.0 International License, which permits use, sharing, adaptation, distribution and reproduction in any medium or format, as long as you give appropriate credit to the original author(s) and the source, provide a link to the Creative Commons licence, and indicate if changes were made. The images or other third party material in this article are included in the article's Creative Commons licence, unless indicated otherwise in a credit line to the material. If material is not included in the article's Creative Commons licence and your intended use is not permitted by statutory regulation or exceeds the permitted use, you will need to obtain permission directly from the copyright holder. To view a copy of this licence, visit http://creativecommons.org/licenses/by/4.0/.

\section{References}

1. Parent-Thirion A, Biletta I, Cabrita J, et al. Eurofound, Sixth European working conditions survey - Overview report (2017 update). Publications Office of the European Union, Luxembourg

2. Vetter C, Devore EE, Ramin CA et al (2015) Mismatch of sleep and work timing and risk of type 2 diabetes. Diabet Care 38(9):1707-1713

3. Zoto E, Cenko F, Doci P et al (2019) Effect of night shift work on risk of diabetes in healthy nurses in Albania. Acta Diabetol 56(7):811-813. https://doi.org/10.1007/s00592-019-01307-8

4. Kervezee L, Cermakian N, Boivin DB (2019) Individual metabolomic signatures of circadian misalignment during simulated night shifts in humans. PLoS Biol 17(6):e3000303. https://doi.org/10. 1371/journal.pbio.3000303

5. Romon M, Nuttens MC, Fievet C et al (1992) Increased triglyceride levels in shift workers. Am J Med 93(3):259-262

6. Lund J, Arendt J, Hampton SM et al (2001) Postprandial hormone and metabolic responses amongst shift workers in Antarctica. J Endocrinol 171(3):557-564

7. Wong PM, Hasler BP, Kamarck TW et al (2015) Social jetlag, chronotype, and cardiometabolic risk. J Clin Endocrinol Metab 100(12):4612-4620

8. Taylor BJ, Matthews KA, Hasler BP et al (2016) Bedtime variability and metabolic health in midlife women: the SWAN sleep study. Sleep 39(2):457-465

9. Li Y, Gao X, Winkelman JW et al (2016) Association between sleeping difficulty and type 2 diabetes in women. Diabetologia 59(4):719-727

10. Reutrakul S, Van Cauter E (2018) Sleep influences on obesity, insulin resistance, and risk of type 2 diabetes. Metabolism 84:56-66

11. Fiorina P, Lattuada G, Ponari O, Silvestrini C, DallAglio P (1996) Impaired nocturnal melatonin excretion and changes 
of immunological status in ischaemic stroke patients. Lancet 347(9002):692-693. https://doi.org/10.1016/s0140-6736(96) 91246-5 (PMID: 8596407)

12. Fiorina P, Lattuada G, Silvestrini C, Ponari O, Dall'Aglio P (1999) Disruption of nocturnal melatonin rhythm and immunological involvement in ischaemic stroke patients. Scand J Immunol 50(2):228-231. https://doi.org/10.1046/j.1365-3083.1999.00579.x (PMID: 10447930)

13. Cardellini M, Menghini R, Luzi A et al (2011) Decreased IRS2 and TIMP3 expression in monocytes from offspring of type 2 diabetic patients is correlated with insulin resistance and increased intima-media thickness. Diabetes 60(12):3265-3270

14. Buysse DJ, Reynolds CF III, Monk TH et al (1989) The Pittsburgh Sleep Quality Index: a new instrument for psychiatric practice and research. Psychiatry Res 28:193-213

15. Lajoie P, Aronson KJ, Day A, Tranmer J (2015) A cross-sectional study of shift work, sleep quality and cardiometabolic risk in female hospital employees. BMJ Open 5:e007327

16. Cardellini M, Rizza S, Casagrande V et al (2019) Soluble ST2 is a biomarker for cardiovascular mortality related to abnormal glucose metabolism in high-risk subjects. Acta Diabetol 56(3):273-280

17. Jakubowicz D, Wainstein J, Landau Z et al (2017) Influences of breakfast on clock gene expression and postprandial glycemia in healthy individuals and individuals with diabetes: a randomized clinical trial. Diabet Care 40:1573-1579. https://doi.org/10.2337/ dc16-2753

18. Yoshida C, Shikata N, Seki S et al (2012) Early nocturnal meal skipping alters the peripheral clock and increases lipogenesis in mice. Nutr Metab (Lond) 9(1):78. https://doi.org/10.1186/ 1743-7075-9-78

19. Pivovarova O, Jürchott K, Rudovich $N$ et al (2015) Changes of dietary fat and carbohydrate content alter central and peripheral clock in humans. J Clin Endocrinol Metab 100(6):2291-2302. https://doi.org/10.1210/jc.2014-3868

20. Morris CJ, Purvis TE, Hu K et al (2016) Circadian misalignment increases cardiovascular disease risk factors in humans. Proc Natl Acad Sci USA 113(10):E1402-E1411. https://doi.org/10.1073/ pnas. 151695311315

21. Pourcet B, Zecchin M, Ferri L et al (2018) Nuclear receptor subfamily 1 group D member 1 regulates circadian activity of NLRP3 inflammasome to reduce the severity of fulminant hepatitis in mice. Gastroenterology 154(5):1449-1464.e20. https://doi.org/ 10.1053/j.gastro.2017.12.019
22. Rizza S, Clementi F, Porzio O et al (2009) Adiponectin isoforms are not associated with the severity of coronary atherosclerosis but with undiagnosed diabetes in patients affected by stable CAD. Nutr Metab Cardiovasc Dis 19(1):54-60. https://doi.org/10.1016/j. numecd.2007.12.001 (Epub 2008 May 9 PMID: 18472407)

23. Rizza S, Longo S, Piciucchi G et al (2020) Carotid intimal medial thickness in rotating night shift is related to IL1 $\beta /$ IL6 axis. Nutr Metab Cardiovasc Dis 30(10):1826-1832. https://doi. org/10.1016/j.numecd.2020.05.028 (Epub 2020 Jun 7 PMID: $32665209)$

24. Takahashi J (2017) Transcriptional architecture of the mammalian circadian clock. Nat Rev Genet 18:164-179. https://doi.org/10. 1038/nrg.2016.150

25. Yang X, Downes M, Yu RT et al (2006) Nuclear receptor expression links the circadian clock to metabolism. Cell 126(4):801-810. https://doi.org/10.1016/j.cell.2006.06.050 (PMID: 16923398)

26. Sato S, Sakurai T, Ogasawara J et al (2014) A circadian clock gene, Rev-erb $\alpha$, modulates the inflammatory function of macrophages through the negative regulation of $\mathrm{Ccl} 2$ expression. $\mathrm{J}$ Immunol 192(1):407-417. https://doi.org/10.4049/jimmunol. 1301982

27. Preitner N, Damiola F, Lopez-Molina L et al (2002) The orphan nuclear receptor REV-ERBalpha controls circadian transcription within the positive limb of the mammalian circadian oscillator. Cell 110:251-260

28. Kim SM, Neuendorff N, Alaniz RC et al (2018) Shift work cycleinduced alterations of circadian rhythms potentiate the effects of high-fat diet on inflammation and metabolism. FASEB J 32(6):3085-3095. https://doi.org/10.1096/fj.201700784R

29. Bal C, Ozturk A, Cicek B et al (2018) The relationship between blood pressure and sleep duration in Turkish children: a crosssectional study. J Clin Res Pediatr Endocrinol 10(1):51-58

30. Mezick EJ, Hall M, Matthews KA (2012) Sleep duration and ambulatory blood pressure in black and white adolescents. Hypertension 59(3):747-752

Publisher's Note Springer Nature remains neutral with regard to jurisdictional claims in published maps and institutional affiliations. 